\title{
ARTICLE
}

\section{A shift of the TOR adaptor from Rictor towards Raptor by semaphorin in C. elegans}

\author{
Akira Nukazuka', Shusaku Tamaki', Kunihiro Matsumoto', Yoichi Oda', Hajime Fujisawa' \& Shin Takagi
}

The target of rapamycin (TOR), a central regulator for cell growth and metabolism, resides in the two functionally distinct complexes TORC1 and TORC2, which are defined by their adaptors Raptor and Rictor, respectively. How the formation of the two TORCs is orchestrated remains unclear. Here we show the control of TOR partnering by semaphorin-plexin signalling in Caenorhabditis elegans. In semaphorin and plexin mutants, TOR-Raptor association decreases whereas TOR-Rictor association increases, concomitantly with TORC1 down- and TORC2 up-regulation. Epidermal defects in the mutants are suppressed by inhibiting TORC2 or reinforcing TORC1 signalling. Conversely, inhibition of TORC1 signalling phenocopies the mutants. Thus, our results indicate that TORC formation is a singularly important step in semaphorin signalling that culminates in diverse outcomes including TORC1-promoted messenger RNA translation and TORC2-regulated cytoskeletal remodelling. 
$\mathrm{S}$ ignals of semaphorins, through their predominant receptors plexins, regulate a wide range of morphogenetic events ${ }^{1}$. Key regulators mediating the signals include cytoskeleton-relevant Rho family GTPases and their effectors such as actin-depolymerizing factor/cofilin ${ }^{2,3}$. Importantly, messenger RNA translation, whose rates are defined by phosphorylation states of translation factors eIF $2 \alpha$ and $4 \mathrm{EBP}^{4}$, is augmented by semaphorins. In vertebrate cultured neurons, semaphorin-induced growth cone responses require rapid mRNA translation through the $4 \mathrm{EBP}-\mathrm{eIF} 4 \mathrm{~F}$ pathway, Recently, our genetic work utilizing the system of 'ray' morphogenesis in Caenorhabditis elegans showed that stimulated translation, which involves phosphorylation control of eIF $2 \alpha$, mediates the semaphorin signal ${ }^{7}$. mRNA species that are translated in response to semaphorins include cofilin and $\mathrm{RhoA}^{6-8}$, suggesting that activation of cytoskeletal regulators, as well as their de novo synthesis, are essential for semaphorin signalling. Mechanisms of the signalling, however, have not been fully elucidated.

The target of rapamycin (TOR), an evolutionarily conserved serine/threonine kinase, is a pivotal regulator for cell growth, morphogenesis and proliferation, which governs and integrates signals from a variety of environmental stimuli ${ }^{9,10}$. TOR resides as a catalytic subunit in two biochemically and functionally distinct multiprotein complexes referred to as TOR complex 1 (TORC1) and TOR complex 2 (TORC2), which are defined by the TOR-associated adaptor proteins Raptor (regulatory associated protein of mTOR) and Rictor (rapamycin-insensitive companion of TOR), respectively ${ }^{11-14}$. An upstream activator of TORC1 is Rheb GTPase ${ }^{15}$, the activity of which can be switched off by the GTPase-activating protein complex TSC1/TSC2 (refs 16-18). Signalling through TORC1 culminates in the promotion of mRNA translation by phosphorylating translational regulators $4 \mathrm{EBP}$ and $\mathrm{S} 6 \mathrm{~K}^{19}$. Functions of TORC2 are less well defined than those of TORC1, but recent work has indicated that TORC2 is involved in remodelling of actin architecture through PKC $\alpha$ and Rac GTPase ${ }^{13,14,20}$. In addition, TORC2 contributes to maintaining cellular viability and size by phosphorylating $\mathrm{AKT} /$ protein kinase $\mathrm{B}^{21-23}$. Rictor is required for TORC2 integrity and substrate specificity, though very little is known about the regulation of TORC2. Another elusive issue is whether and how the two TORCs integrities are orchestrated in vivo.

In this study, we report a new link between the semaphorin input and TOR partnering. Through genetic analyses in C. elegans, we demonstrate that semaphorin activates TORC1 and simultane- ously inhibits TORC2 by promoting a shift of the TOR adaptor from Rictor towards Raptor. Furthermore, not only activation of TORC1 but inhibition of TORC2 is also required for semaphorin-induced epidermal cellular morphogenesis. Together, we propose that TOR integrates and transduces the upstream semaphorin signal by acting as a central commander that orchestrates multiple downstream cascades.

\section{Results}

Loss of Rictor suppresses the defects in semaphorin mutants. In C. elegans, signalling of membrane-bound semaphorins (SMP-1 \& SMP-2; SMP) through plexin (PLX-1) regulates the arrangement of rays: bilateral nine-paired sensilla found in a male tail ${ }^{24,25}$. Each protruding ray consists of three cells including a support cell and two neuronal cells, all of which originate from an epidermal cellular cluster $^{26}$. At the early third larval stage (L3 stage), several rounds of epidermal cell divisions in a tail generate nine sets of clusters as well as extra hypodermal Rn.p cells $(n=1-9)$. One major role of the SMP-PLX-1 signal is to posteriorly shift the boundary between R1.p and R2.p at the late L3 stage ${ }^{24}$. Because the ray 1-differentiating cluster (cluster 1) is destined to be located at the R1.p-R2.p boundary ${ }^{27}$, its posterior shift allows for the location of cluster 1 adjacent to cluster 2. This is how, in wild-type adults, ray 1 is juxtaposed to ray 2 . By contrast in $p l x-1$ and smp- 1 smp-2 mutants, the R1.p-R2. $\mathrm{p}$ boundary fails to undergo the posterior shift and thus cluster 1 remains situated anteriorly (Fig. 1a), which consequently leads to anterior displacement of ray 1 at the adult stage (Fig. 1b). The ray 1 phenotype in plx-1 mutants is markedly rescued by expressing wild-type $p l x-1$ under the promoter of lin-32 gene (lin-32p), a ray lineage-specific driver ${ }^{7,28}$ (Fig. 1c).

To investigate the downstream events of SMP signalling, we conducted a screen for suppressors of $p l x-1$ mutation and isolated a recessive allele, $n c 41$, which almost fully suppressed the ray phenotype in mutants (Fig. 1a-c). No abnormality was found in the ray configuration in nc4l single mutants (Supplementary Table S1). nc41 was mapped to the right arm of linkage group II and was shown through the sequencing to be a 470-bp deletion within rict-1 gene (Supplementary Fig. S1a and b), which encodes a protein weakly orthologous to Rictor ${ }^{29,30}$. Nucleotides 3,538-3,586 (49 bases) corresponding to part of the 12th exon are deleted in the nc41-type rict-1 transcript, which produces a nonsense frame-shift leading to the premature opal codon at the predicted 1,200 th codon

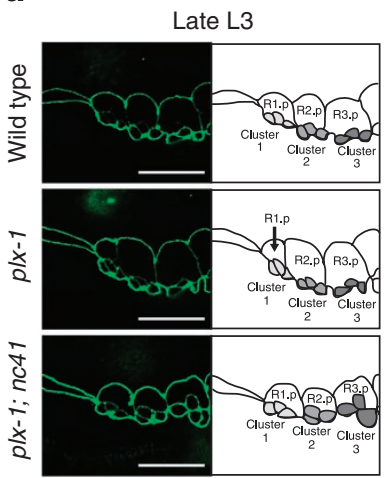

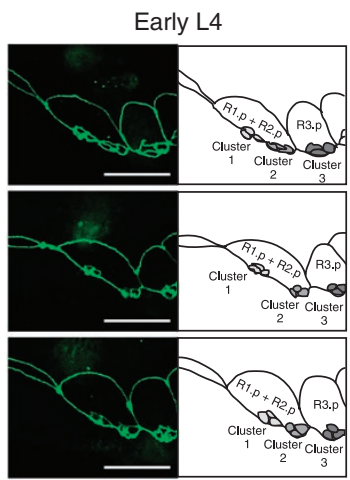

b

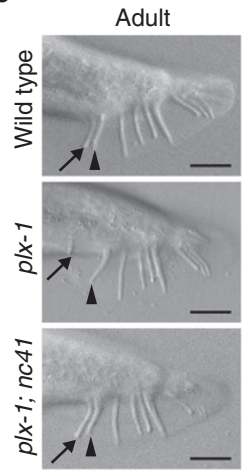

C $(\%)$

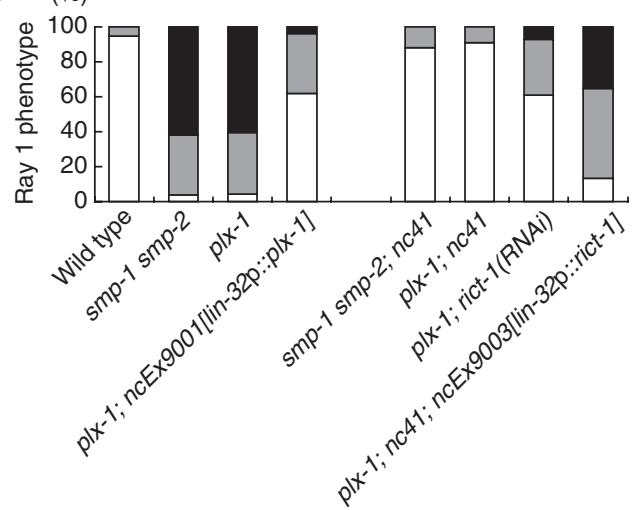

Figure 1 | Loss of rict-1 function suppresses the ray 1 phenotype in plx-1 and smp-1 smp-2 mutants. (a) Configurations of epidermal ray precursor cells visualized with ajm-1::gfp in a wild-type, a plx-1 mutant, and a plx-1; rict-1(nc41) mutant animal at the late L3 stage and the early L4 stage. Schematic drawings illustrating the contours of cells are also given. Anterior is left, and dorsal is top. Scale bars, $10 \mu \mathrm{m}$. (b) Nomarski images of adult male tails. Ray 1 (arrow), which is located close to ray 2 (arrowhead) in a wild type, is located abnormally at the anterior position in a plx- 1 mutant, but it is reverted to the normal position in a plx-1; rict-1(nc41) mutant animal. Anterior is left, and dorsal is top. Scale bars, $10 \mu \mathrm{m}$. (c) Penetrance of the ray 1 phenotype in adults of the indicated genotypes. For the quantitative evaluation, the ray 1 phenotypes are categorized into either Level 1 (normal), Level 2 (mildly defective), or Level 3 (severely defective), which are represented by white, grey or black segment of each bar, respectively. $n=215-234$ for each genotype. 
a
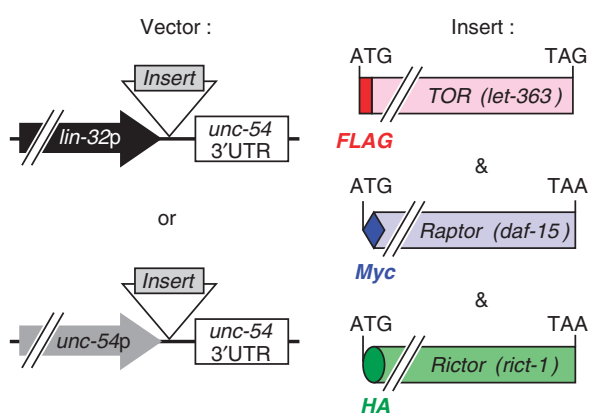

b

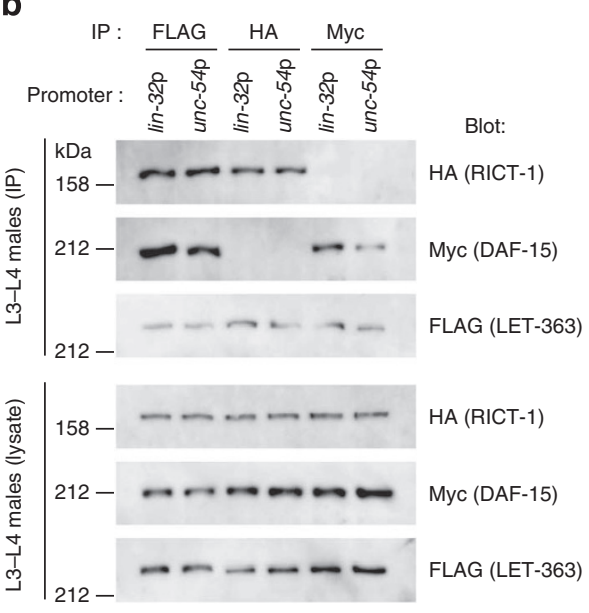

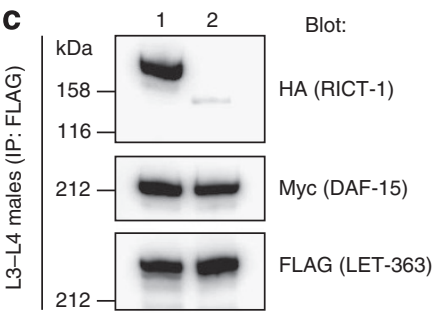

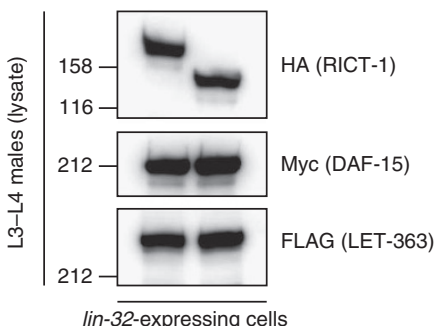

Figure 2 | DAF-15 and RICT-1 define TORC1 and TORC2, respectively. (a) Schemes of expression constructs for FLAG-tagged TOR (LET-363), Myctagged Raptor (DAF-15), and HA-tagged Rictor (RICT-1), all of which are placed under either the lin-32 promoter or the unc-54 promoter. (b) Mutually exclusive association of DAF-15 and RICT-1 with LET-363. Transgenic L3-L4 males expressing epitope-tagged RICT-1, DAF-15 and LET-363 proteins under the lin-32p (ncEx9005) or the unc-54p (ncEx9007) were lysed, and proteins were immunoprecipitated with antibodies against the indicated tags. The immunocomplex (IP) and lysates were analysed by immunoblotting. (c) Abolished association of nc41-type RICT-1 with LET-363. Transgenic L3-L4 males expressing wild-type RICT-1 (lane 1: ncEx9005) or nc41-type RICT-1 (lane 2: ncEx9008) together with DAF-15 and LET-363 under the lin-32p were lysed, and FLAG :: LET-363 proteins were immunoprecipitated. The FLAG-precipitated IP and lysates were analysed by immunoblotting.

(Supplementary Fig. S1c). Knockdown of rict-1 by RNAi suppressed the plx-1 mutant phenotype, whereas wild-type rict-1 expression under the lin-32p reversed the suppression by $n c 41$ (Fig. 1c). Thus, we concluded that $n c 41$ is a loss-of-function rict- 1 allele, which is responsible for suppressing the ray 1 phenotype in $p l x-1$ and $s m p-1$ smp-2 mutants.

C. elegans Raptor and Rictor define TORC1 and TORC2. In other organisms, Rictor binds TOR to form a multiprotein complex TOR complex 2 (TORC2) $^{13,14}$. Independent of TORC2, TOR also forms the complex TORC1 with its defining component Raptor ${ }^{11,12}$.

To examine TORC formation in C. elegans, we cloned complementary DNAs for TOR (let-363) ${ }^{31}$, Raptor (daf-15) $)^{32}$ and Rictor (rict-1), and tagged them at their $5^{\prime}$ ends with FLAG, Myc and HA oligonucleotides, respectively. Then, we generated a transgenic line expressing LET-363, DAF-15 and RICT-1 placed under the lin-32p, together with wild-type $p l x-1 \mathrm{cDNA}$ placed under a heat-shock promoter ( $h s p$ ) (Fig. 2a). The resultant line expressing these transgenes seems to be physiologically balanced; no interference with the ray 1 phenotype was observed in the background of wild-type, plx-1 and smp-1 smp-2 mutants (Supplementary Table S1). We also generated a line expressing epitope-tagged LET-363, DAF-15 and RICT-1 under the promoter of $u n c-54$, a gene specific to body wall muscles, where plx-1 expression does not overlap ${ }^{24}$ (Fig. 2a). After retrieval of transgenic males at L3-L4 stages, FLAG::LET-363-, Myc::DAF-15- or HA :: RICT-1-containing complexes were immunoprecipitated with antibodies against their respective epitope tags and the immunocomplex (IP) was analysed. In both ray precursor cells (lin-32-positive) and muscle cells (unc-54-positive), the LET-363 IP contained both DAF-15 and RICT-1 (Fig. 2b). On the other hand, the DAF-15 IP contained LET-363 but not RICT-1, whereas the RICT-1 IP contained LET-363 but not DAF-15 (Fig. 2b). Thus, similar to Raptor and Rictor in other organisms, C. elegans DAF-15 and RICT-1 also define TORC1 and TORC2, respectively. In another line expressing nc41-type RICT-1 together with LET-363 and DAF-15 under the lin-32p, LET-363-RICT-1 association was largely abolished (Fig. 2c), indicating that TORC2 signalling is blocked by rict-1(nc41) deletion.
Active TORC1 and inactive TORC2 mediate semaphorin signal. To address whether perturbed TORC formation by rict- 1 mutation is responsible for the suppression of the ray phenotype in $p l x-1$ and smp-1 smp-2 mutants, we knocked-down C. elegans LST8 (CeLST8), whose mammalian orthologue is reportedly essential for signalling of TORC2 but is dispensable for that of TORC1 (ref. 23). CeLST8 knockdown markedly suppressed the plx-1 mutant phenotype (Fig. 3a). Furthermore, the knockdown obliterated LET-363RICT-1 association and, to our surprise, increased LET-363-DAF-15 association (Fig. 3b). We also found that rict-1 knockdown resulted in an increase in LET-363-DAF-15 association, and daf-15 knockdown, likewise, resulted in an increase in LET-363-RICT-1 association, suggesting a competitive relationship between DAF-15 and RICT-1 for binding to LET-363 (Supplementary Fig. S2). Thus, we presumed that, in addition to decreased TORC2 signalling, increased TORC1 signalling might be involved in bypassing the SMP signal. To examine the possible contribution of increased TORC1 to suppression, we overexpressed DAF-15 under the lin-32p, and found that the phenotypes in $p l x-1$ and $s m p-1 s m p-2$ mutant were partially suppressed (Fig. 3c). We also tested whether loss of putative TORC1 inhibitors had a similar suppressive effect on the plx-1 mutant phenotype as DAF-15 overexpression did. LKB1 exists in a heterotrimeric complex with two accessory proteins, Ste20-related adaptor protein (STRAD) and MO25. This complex phosphorylates and activates AMPK, thereby inhibiting the TORC1 pathway ${ }^{33}$. Loss of LKB1 (par-4), STRAD (strd-1), catalytic $\alpha$ subunit of AMPK (aak-1 $\& a a k-2)$ and $\beta$ subunit of AMPK (aakb-1) $)^{34-36}$ partially suppressed the plx-1 mutant phenotype (Fig. $3 \mathrm{~d}$ ). These results suggest that, in addition to TORC2 inhibition, TORC1 activation partially contributes to bypassing the SMP signal.

Conversely, we analysed the effects of TORC1 inhibition and TORC2 activation on the ray morphogenesis. Knockdown of TORC1 component genes, let-363 and daf-15, by a standard RNAi protocol causes developmental arrest ${ }^{31,32}$ and prevents analysis of the ray morphology. As an alternative strategy, ray-specific RNAi was exploited (Methods). We found that ray-specific RNAi against let-363 and daf-15 caused the ray phenotype to be as penetrant as ray-specific plx-1 RNAi (Fig. $3 \mathrm{e}-\mathrm{g}$ ), but did not suppress the 


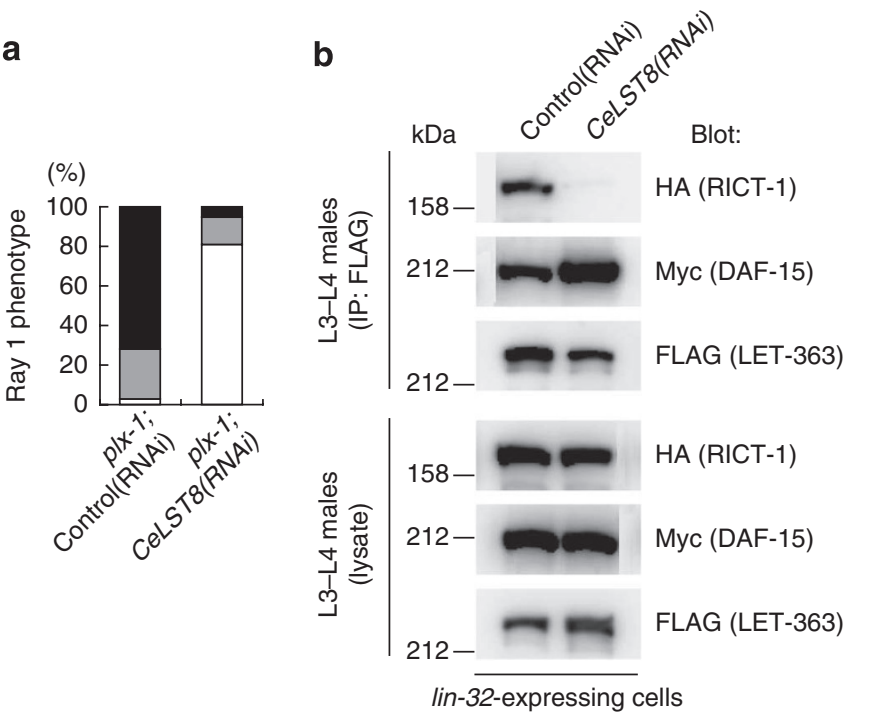

e

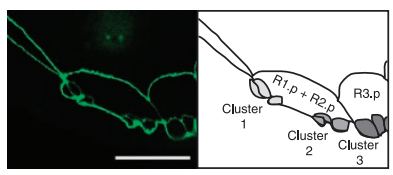

f

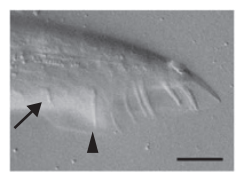

C

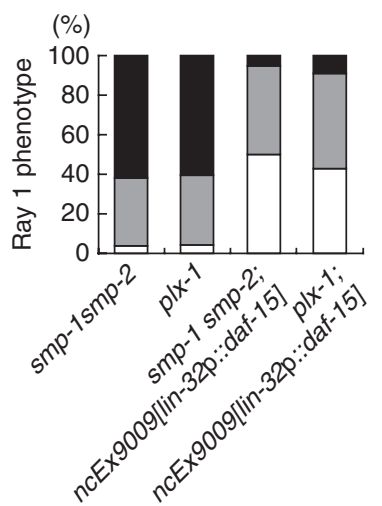

d

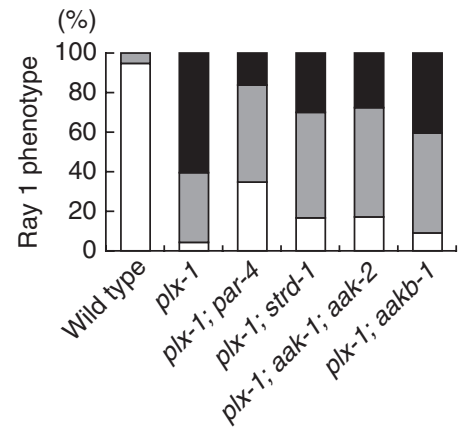

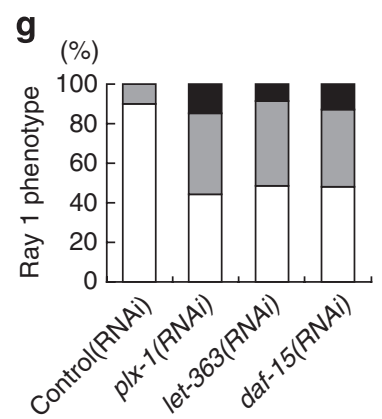

Figure 3 | TORC2 inhibition and/or TORC1 activation bypass the SMP signal. (a) Quantitative ray 1 phenotype caused by CeLST8 knockdown in the p/x-1 mutant background. (b) Transgenic L3-L4 males (ncEx9005) were subjected to either control or CeLST8 RNAi, and then lysed. FLAG ::LET-363 proteins in the lysate were immunoprecipitated, followed by analysis of IP and lysates. (c) Quantitative ray 1 phenotype in animals overexpressing DAF-15 under the lin-32p in the background of $p / x-1$ or smp-1 smp-2 mutants. (d) Quantitative ray 1 phenotypes caused by loss of LKB1 (par-4), STRAD (strd-1), AMPK $\alpha$ (aak-1 $\& a a k-2)$ or AMPK $\beta$ (aakb-1) in the background of plx-1 mutants. (e) Epidermal ray precursor cells visualized with ajm-1::gfp in a daf-15(RNAi) male at the early L4 stage. Anterior is left, and dorsal is top. Scale bar, $10 \mu \mathrm{m}$. (f) Nomarski image of a tail in a daf-15(RNAi) adult male. An arrow indicates ray 1; an arrowhead, ray 2. Anterior is left, and dorsal is top. Scale bar, $10 \mu \mathrm{m}$. (g) Quantitative ray 1 phenotype in animals subjected to ray-specific RNAi against plx-1, let-363 and daf-15. For $\mathbf{a , c}, \mathbf{d}, \mathbf{g}$ : for the quantitative evaluation, the ray 1 phenotypes are categorized into either Level 1 (normal), Level 2 (mildly defective), or Level 3 (severely defective), which are represented by white, grey or black segment of each bar, respectively $n=148-234$ for each genotype.

plx-1 mutant phenotype (Supplementary Table S1). Likewise, a less pronounced but similar phenotype was observed by the lin-32pdriven RICT-1 overexpression, which presumably increases TORC2 (Supplementary Table S1). Thus, these results suggest that TORC1 cooperates with SMP signalling whereas TORC2 antagonizes it.

Semaphorin shifts the TOR adaptor from Rictor to Raptor. Our results led us to speculate that SMP regulates the assembly of the two TORCs. To investigate this possibility, we compared the amount of DAF-15 and that of RICT-1 complexed with LET-363 in lin-32expressing ray precursor cells between wild types, $p l x-1$ mutants and smp-1 smp-2 mutants. We retrieved transgenic males at L3-L4 stages, during which ray morphogenesis is controlled by the SMP signal, so as to detect the TORC formation during the SMP-regulated ray morphogenesis. In mutants, the amount of LET-363-bound RICT- 1 increased by about $240 \%$, whereas that of LET-363-bound DAF-15 decreased by about $60 \%$ of wild types (Fig. $4 \mathrm{a}$; Supplementary Fig. S3). Restoration of plx-1 expression by heat shock rescued the perturbed TORC formation in plx-1 mutants, whereas it failed to rescue that in $s m p-1 s m p-2$ mutants (Fig. $4 \mathrm{a}$ ), probably because of the incapability of PLX-1 alone to transduce the signal in the absence of its ligands. As the total amounts of RICT-1, DAF-15 and
LET-363 in the whole lysates were indistinguishable among genotypes (Fig. 4a), it is indicated that the SMP signal through PLX-1 serves to promote a shift of the LET-363-associated adaptor from RICT-1 towards DAF-15. By contrast in unc-54-expressing muscle cells, where PLX-1 expression is absent, significant difference was not observed in the amount of LET-363-bound DAF-15 and RICT-1 between wild types and plx-1 mutants (Fig. 4b), confirming the importance of SMP-PLX-1 signalling on TOR partnering. To test further the SMP-regulated TORC formation, we utilized the system of SMP signal reinforcement that can be triggered by simultaneous expression of $s m p-1$ and $p l x-1$ by heat shock, which was previously shown to quickly enhance SMP signalling ${ }^{7}$. Both in ray precursor cells and muscle cells, reinforcement of the SMP signal resulted in a further decrease in LET-363-RICT-1 and an increase in LET363-DAF-15 association (Fig. 4c), indicative of an acute effect of the SMP signal on the TORC formation.

To test whether decreased TORC1 and increased TORC2 in plx-1 mutant ray precursor cells are associated with changes in their activities, we monitored a phosphorylation state of a TORC1 substrate, $4 \mathrm{EBP}^{19}$, and that of a TORC2 substrate, $\mathrm{PKC} \alpha^{13,23}$. As SPN-2, a putative counterpart of the translational repressor 4EBP1 (ref. 37), and four PKC proteins encoded by $p k c-1$ (PKCe), pkc-2 (PKC $\alpha)$, 
a
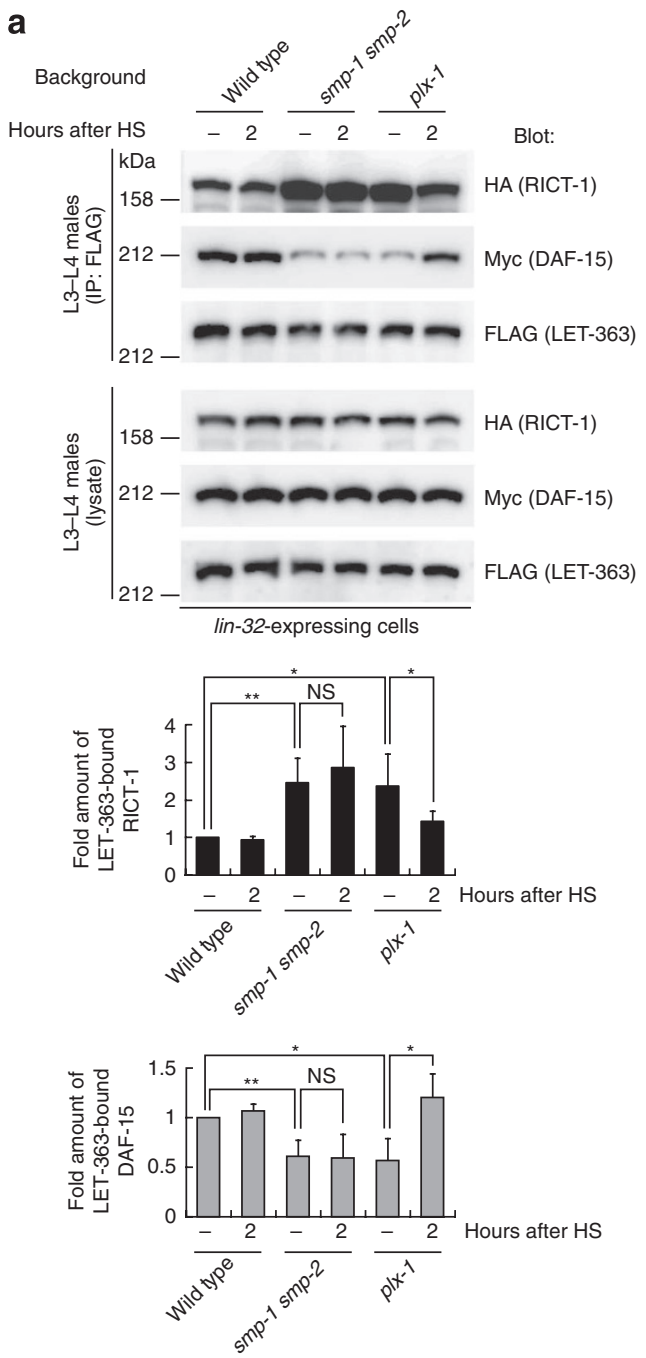

b
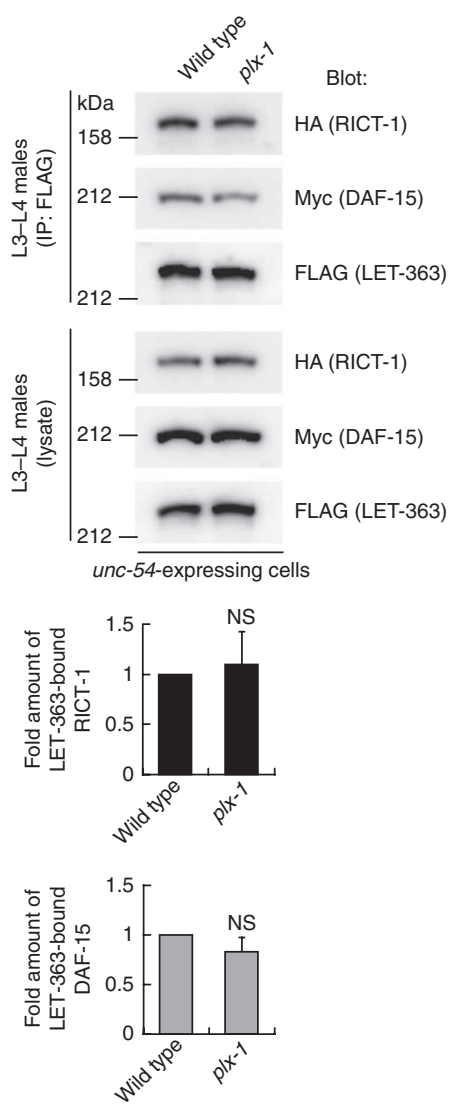

c
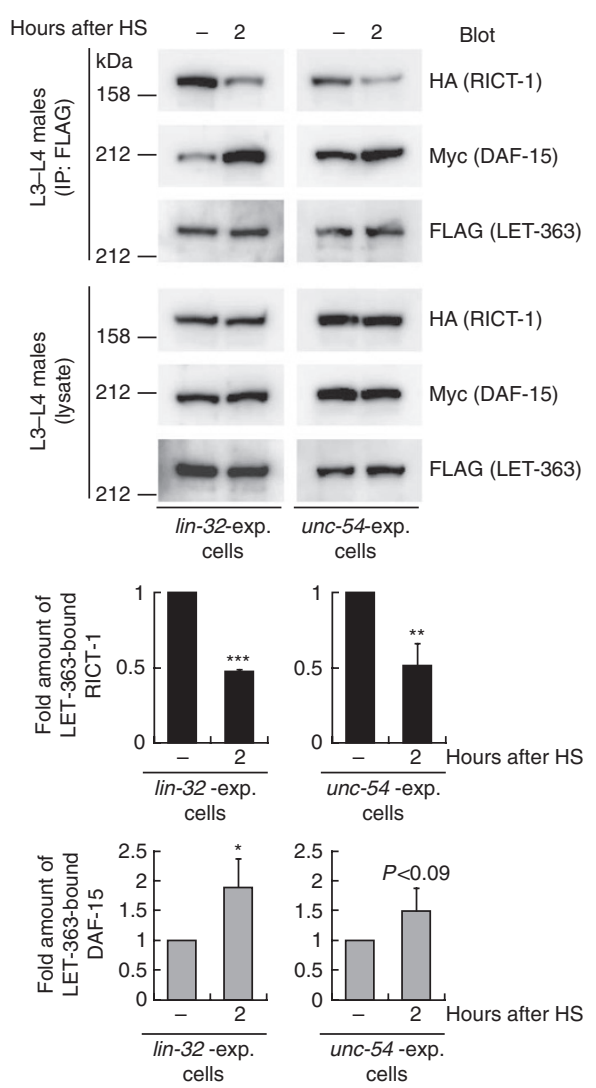

Figure 4 | The SMP signal promotes a shift of the LET-363-associated adaptor from RICT-1 towards DAF-15. (a) TORC1 and TORC2 formations in wild-type, plx-1 and smp-1 smp-2 mutant ray precursor cells. Transgenic L3-L4 males (ncEx9005) in the indicated genetic backgrounds, which carry three TORC component genes under the lin-32p together with plx-1 under hsp ( $h s p:: p / x-1$ ), were either untreated ( - ) or treated with brief heat-shock and collected two hours after heat-shock (2). FLAG ::LET-363 proteins in the lysate were immunoprecipitated, followed by analysis of IP and lysates. The intensity of co-immunoprecipitated HA ::RICT-1 (black bars) or Myc::DAF-15 (grey bars) normalized by FLAG ::LET-363 in IP is shown in the graphs. Shown are the means \pm s.e.m. of four independent experiments. (b) TORC1 and TORC2 formations in unc-54-expressing muscle cells in wild-type and plx-1 mutants, which were analysed with a line, ncEx9007. The intensity of co-immunoprecipitated HA::RICT-1 (black bars) or Myc::DAF-15 (grey bars) normalized by FLAG ::LET-363 in IP is shown in the graphs. Shown in the graphs are the means \pm s.e.m. of four independent experiments. (c) TORC1 and TORC2 formations under reinforced SMP signalling. The same protocol was employed as in a, except for that the lines carry both $h s p:: s m p-1$ and $h s p:: p / x-1$ transgenes. ncEx9011 was utilized to analyse the TORC formations in lin-32-expressing cells; ncEx9012, in unc-54-expressing cells. The intensity of coimmunoprecipitated HA ::RICT-1 (black bars) or Myc::DAF-15 (grey bars) normalized by FLAG ::LET-363 in IP is shown in the graphs. Shown in the graphs are the means \pm s.e.m. of three independent experiments. One asterisk indicates $P<0.05$; two asterisks, $P<0.005$; three asterisks, $P<0.001$ for all panels. Paired $t$-test was carried out to calculate the $P$-values. NS, not significant $(P>0.05)$.

$p k c-3(\mathrm{PKC} l)$ and tpa-1 (PKC $\delta)^{38}$, cannot be recognized by commercially available antibodies, an alternative strategy to monitor the C. elegans TORC activities was adopted by utilizing a line expressing human 4EBP1 (h4EBP1) or rat PKC $\alpha$ ( $\mathrm{rPKC} \alpha)$ tagged with FLAG and placed under the lin-32p. These xenogeneic molecules appeared to serve as substrates for C. elegans TORCs, because let-363 and daf-15 knockdowns abolished h4EBP1 phosphorylation (P-h4EBP1) whereas let-363 knockdown and rict-1 mutation abolished P-rPKC $\alpha$ (Fig. 5a). In $p l x-1$ mutants, P-h4EBP1 reduced by about $60 \%$, whereas P-rPKC $\alpha$ increased by about $150 \%$ of wild types (Fig. 5a), which seemingly correlates with decreased TORC1 and increased TORC2 formations in mutants. Taken together, these results indicate that SMP signalling activates TORC1 whereas it inactivates TORC2 by shifting the LET-363-associated adaptor from RICT-1 to DAF-15.

Semaphorin signalling through active TORC1 and inactive TORC2. Previously, we showed that knockdown of ifg-1 (eIF4G; a component of eIF4F complex) phenocopies plx-1 and smp-1 smp-2 mutants, whereas knockdown of spn-2 (4EBP; a translational repressor) partially suppresses the $p l x-1$ mutant phenotype (Fig. 5b) ${ }^{7}$. Taking these findings together with our present result of reduced P-h4EBP1 in $p l x-1$ mutants, we reasoned that increased and thus upregulated TORC1 mediates the SMP signal by repressing SPN-2 and activating translation. However, loss of S6K (rsks1), another putative TORC1 substrate ${ }^{19}$, did not cause ray defects 
a
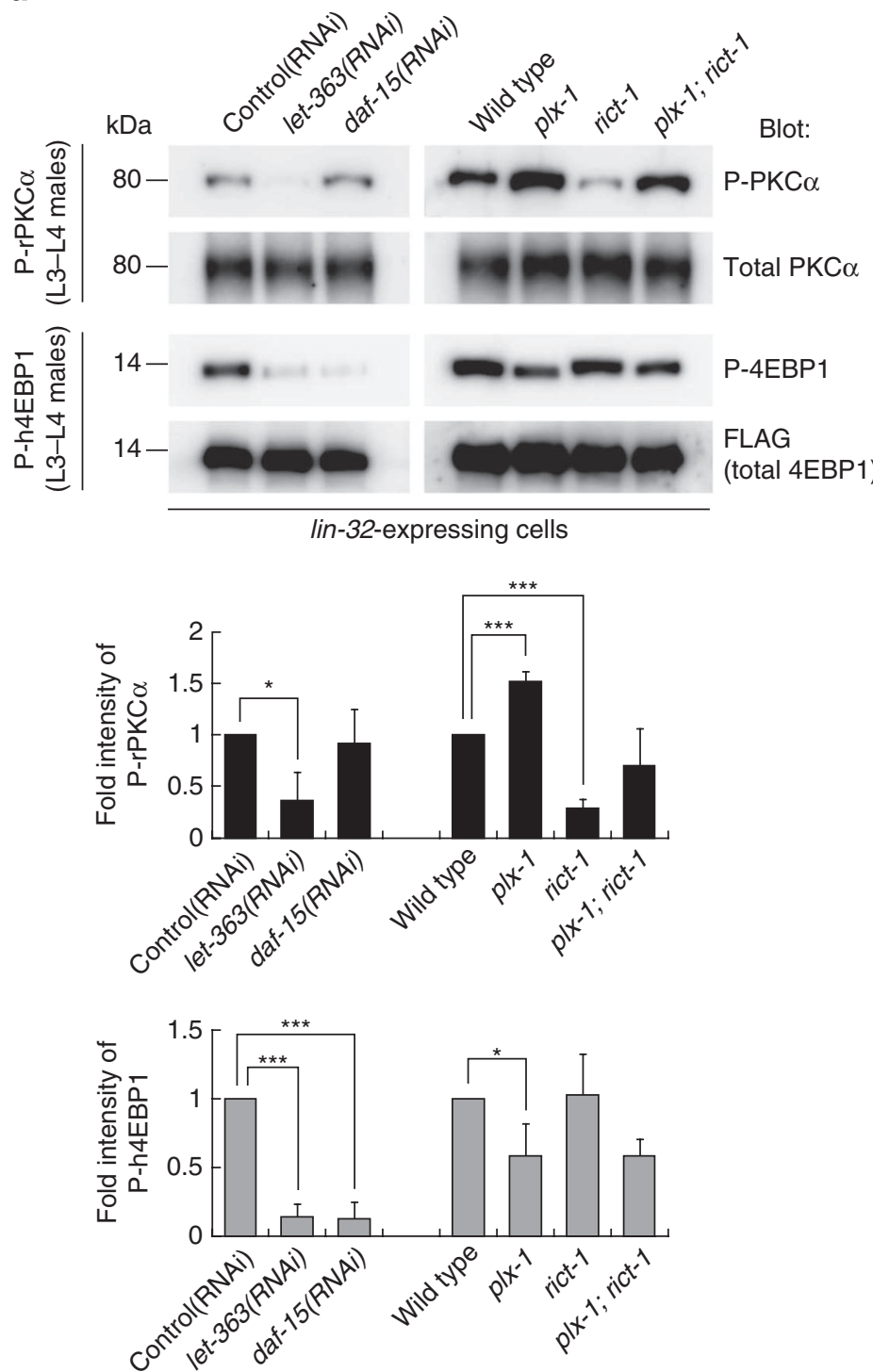

b $(\%)$
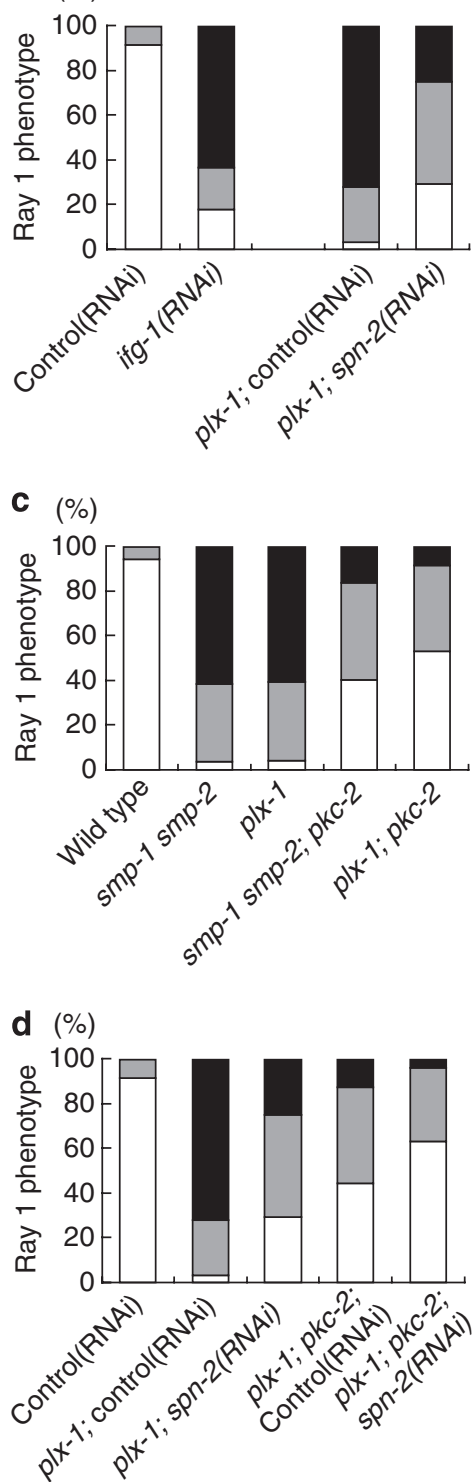

Figure 5 | SMP signalling necessitates both TORC1 activation and TORC2 inactivation. (a) Phosphorylation of xenogeneically expressed rat PKC $\alpha$ ( $r$ PKC $\alpha$ ) and human 4EBP1 (h4EBP1) in lin-32-expressing cells were used to assess the activities of TORC2 and TORC1, respectively. L3-L4 males in the indicated genetic background carrying either the lin-32p::FLAG ::rPKC $\alpha$ transgene (ncEx9014) or the lin-32p::FLAG::h4EBP1 transgene (ncEx9013) were lysed, and analysed for their phosphorylation states by immunoblotting. Black bars represent normalized P-rPKC $\alpha$; grey bars, normalized P-h4EBP1. Shown in the graphs are the means \pm s.e.m. of three independent experiments. One asterisk indicates $P<0.05$; three asterisks, $P<0.001$. Paired $t$-test was carried out to calculate the $P$-values. (b) Quantitative ray 1 phenotypes in wild-type adults subjected to ifg- 1 RNAi and $p / x-1$ mutant adults subjected to spn- 2 RNAi. (c) Quantitative ray 1 phenotypes caused by loss of pkc-2 function in the genetic background of plx-1 or smp-1 smp-2 mutants. (d) Quantitative ray 1 phenotypes of spn-2 and pkc-2 double knockdown in the genetic background of plx-1 mutants. For $\mathbf{b}$ - $\mathbf{d}$ : for the quantitative evaluation, the ray 1 phenotypes are categorized into either Level 1 (normal), Level 2 (mildly defective), or Level 3 (severely defective), which are represented by white, grey or black segment of each bar, respectively. $n=105-234$ for each genotype.

(Supplementary Fig. S4a). This result rules out possible contribution of S6K to SMP signalling, and is consistent with a previous report arguing against $C$. elegans $\mathrm{S} 6 \mathrm{~K}$ as the TOR downstream mediator ${ }^{31}$.

Next, to investigate the biological relevance of SMP-induced TORC2 downregulation, we disrupted PKC, a putative TORC2 substrate $^{13,23}$. Striking but not full suppression of the $p l x-1$ and $s m p-1$ smp-2 mutant phenotype was observed by $p k c-2$ and $p k c-3$ deficiency (Fig. 5c; Supplementary Fig. S4b). Loss of the other PKC isoforms, $p k c-1$ and $t p a-1$, did not significantly affect the $s m p-1 s m p-2$ mutant phenotype (Supplementary Fig. S4b). Thus, inhibition of PKC $\alpha$ and possibly $\mathrm{PKC} \imath$ caused by TORC2 downregulation is suggested to be another means by which SMP signalling controls the proper ray morphogenesis. Intriguingly, simultaneous inhibition of $s p n-2$ and $p k c-2$ suppressed the $p l x-1$ mutant phenotype more strikingly than either single inhibition (Fig. 5d), supporting an idea that SMP signalling requires both TORC1 activation and TORC2 inactivation. We also analysed roles of AKTs $(a k t-1 \& a k t-2)^{39}$, well-defined TORC2 substrates ${ }^{21}$, and FoxO (daf-16), a putative AKT effector ${ }^{39}$, on SMP-mediated ray positioning. Unexpectedly, their deficiencies neither caused the ray 1 phenotype nor suppressed the plx-1 mutant phenotype (Supplementary Fig. S4c), excluding the idea that AKT-FoxO signalling is involved in the SMP-TORC2 pathway. 

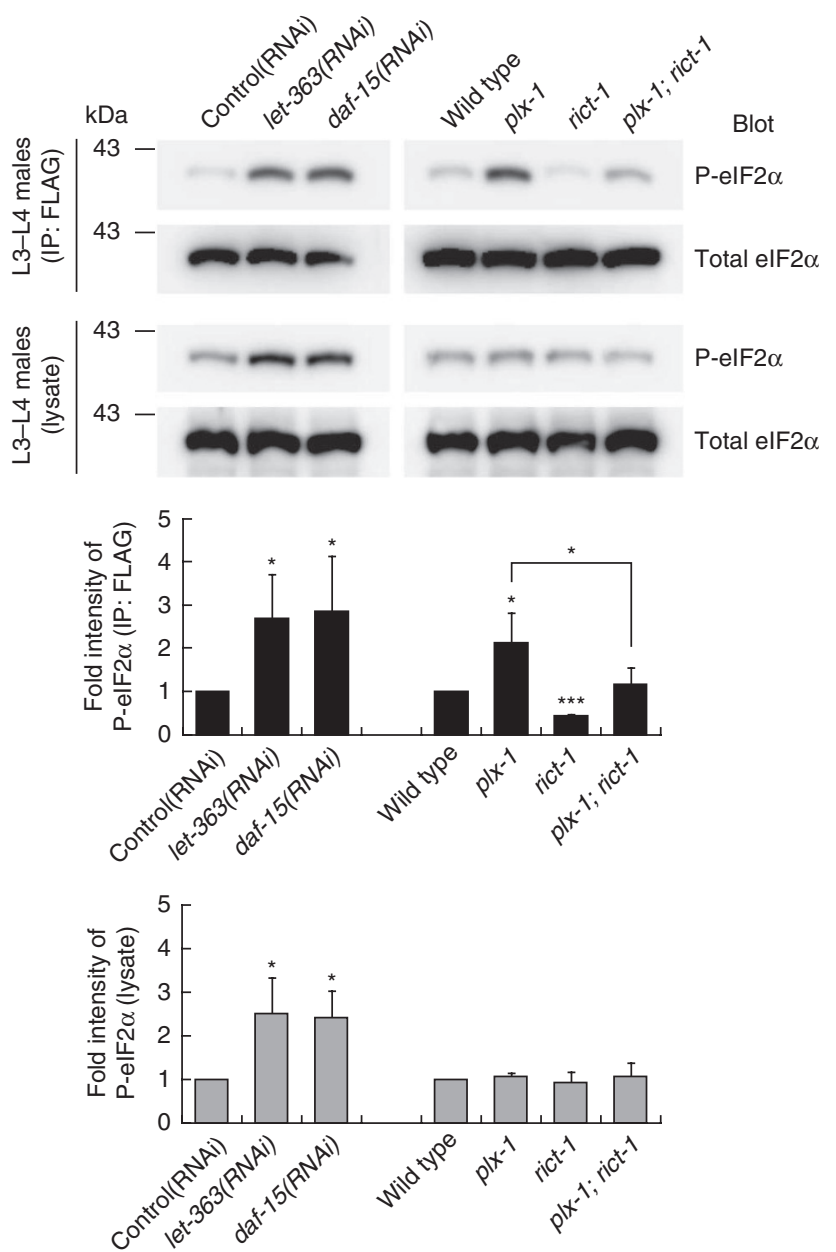

Figure 6 | elF2 $\alpha$ phosphorylation is influenced by the TOR complex signal. L3-L4 males carrying the lin-32p::elF2 $\alpha:: F L A G$ transgene (ncEx9002) were lysed, and FLAG-tagged elF2 $\alpha$ proteins were immunoprecipitated and subjected to immunoblot analysis with antibodies against total elF $2 \alpha$ or phospho-elF $2 \alpha$. Black bars represent P-elF2 $\alpha$ in IP; grey bars, in lysate. Shown in the graphs are the means of normalized P-elF2 $\alpha \pm$ s.e.m. of three independent experiments. One asterisk indicates $P<0.05$; three asterisks, $P<0.001$. Paired $t$-test was carried out to calculate the $P$-values.

As previously suggested ${ }^{30}$, AKTs may not be the primary effectors of TORC2 in C. elegans.

Finally, we examined whether the activity of eIF2, another translation factor that participates in translation initiation and has been shown to mediate the SMP signal ${ }^{7}$, is also under the control of TORCs. We observed higher phosphorylation of its $\alpha$ subunit $(\mathrm{eIF} 2 \alpha)$, representing diminished global translation, in let-363 and daf-15 knockdowns similar to plx-1 mutants (Fig. 6). Thus, through an as yet unknown mechanism, TORC1 seems to serve as a central mediator of SMP-stimulated mRNA translation through eIF2.

\section{Discussion}

By contrast to extensive understandings on regulation of TORC1, that of TORC2 has remained poorly characterized. Here we provide the genetic demonstration of an aspect of TORC2 regulation in C. elegans. We showed that TORC2 is downregulated by the morphogenetic semaphorin signal, and this coincides with TORC1 upregulation, which are based on semaphorin-induced concerted alteration in biochemical assemblies of the two TORCs.

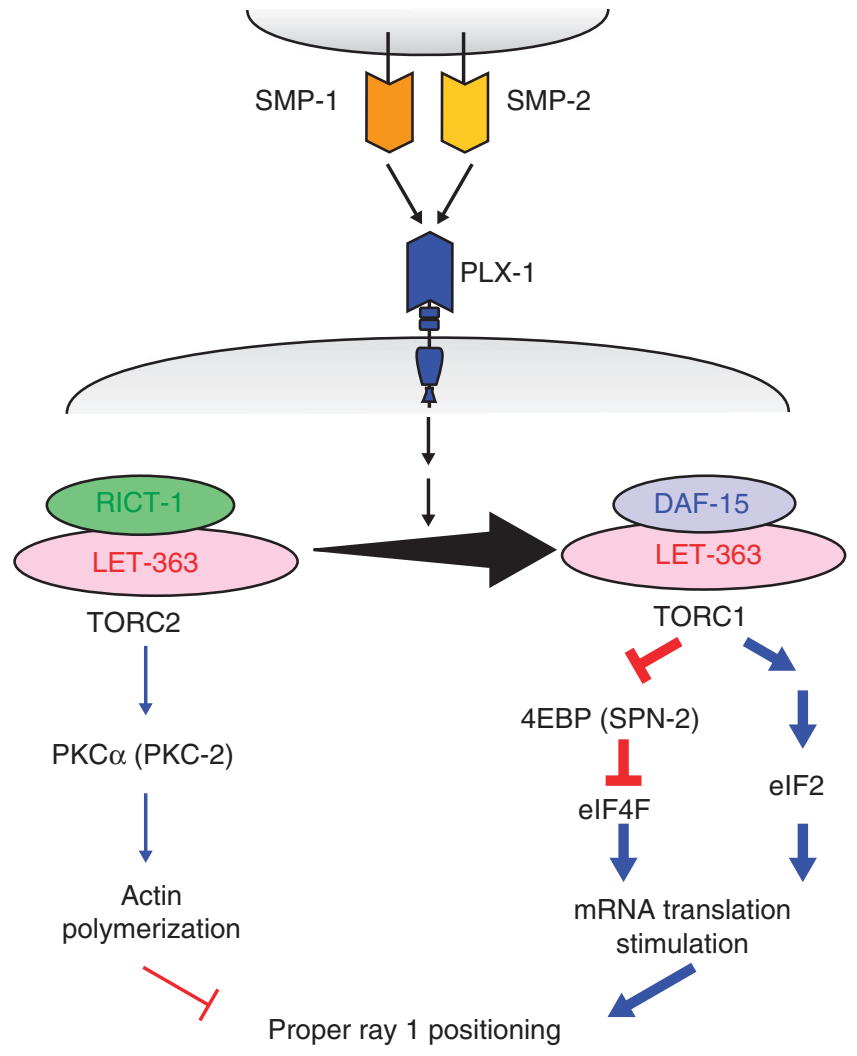

Figure 7 | A model illustrating semaphorin signalling through TOR leading to morphogenetic changes in epidermal cells. The semaphorin signal through plexin promotes a shift of the LET-363 adaptor from RICT-1 towards DAF-15. Increase in the level of TORC1 culminates in translation stimulation of mRNAs. This involves activation of elF4F by inhibiting its negative regulator $4 \mathrm{EBP}$ through phosphorylation, as well as activation of elF2 by reducing phosphorylation of elF $2 \alpha$. Concomitantly, decrease in the level of TORC2 reduces the level of phospho-PKC $\alpha$, which probably leads to destabilization of actin cytoskeleton.

Our present study began with isolation of Rictor deficiency as a suppressor of the plexin mutant ray phenotype, providing us with a clue to the semaphorin action on TOR. Akin to Rictor deficiency, Raptor overexpression suppressed the semaphorin and plexin mutant phenotype. Conversely, animals deficient in Raptor and TOR, as well as animals overexpressing Rictor, were phenotypically similar to semaphorin and plexin mutants, indicating that TORC1 cooperates with whereas TORC2 antagonizes semaphorin signalling. A key finding is that Raptor-TOR association was diminished whereas Rictor-TOR association was facilitated in semaphorin and plexin mutants, along with TORC1 down- and TORC2 up-regulation. Moreover, reinforcement of semaphorin input in the wild-type background was sufficient to quickly diminish TORC2 while facilitating TORC1 assembly. Since the total amounts of Rictor and Raptor were indistinguishable, irrespective of semaphorin input, our results indicate that semaphorin post-translationally shifts the TOR adaptor from Rictor towards Raptor (Fig. 7).

What are the molecular consequences of alteration in the ratio of TORC1 to TORC2 by semaphorin? Activated TORC1 augments translation by phosphorylating and antagonizing a translation inhibitor $4 \mathrm{EBP}^{19}$. We observed decreased P-h4EBP1 in plexin mutants, which appeared relevant to decreased TORC1. In addition, 4EBP knockdown suppressed the ray defect in plexin mutants. Thus, we propose that semaphorin stimulates translation through 
TORC1-mediated negative regulation on 4EBP. This is consistent with our previous finding of a role for semaphorin in translation stimulation through another translation factor, eIF $2 \alpha^{7}$. Moreover, compelling evidence came from studies on vertebrate neurons, which showed that semaphorin triggers 4EBP phosphorylation and stimulates translation ${ }^{5,6}$. A TORC1 inhibitor rapamycin blocks the semaphorin-induced growth cone responses ${ }^{5}$, in agreement with our finding that Raptor and TOR deficients phenocopied semaphorin and plexin mutants. Another intriguing observation is that TORC1 inhibition (TOR and Raptor knockdowns) not only decreased Ph4EBP1 but elevated P-eIF2 $\alpha$, implicating a cross-talk between the two regulatory pathways for translation by TORC1. This is reminiscent of reports that rapamycin inhibits TAP42 phosphatase complex-associated TORC1, resulting in translation repression through increased eIF $2 \alpha$ phosphorylation ${ }^{40,41}$. Therefore, we postulate that the semaphorin signal is integrated and diverged by TORC1 into activation of eIF2 and eIF4F systems, which subsequently converges to promote translation.

Growing evidence suggests that TORC2 modulates cytoskeletonbased events, including cell morphogenesis and migration ${ }^{13,14,20,42,43}$. These events are mediated in part by Rho GTPases and PKC, which eventually induce actin polymerization. Here we report PKC $\alpha /$ $\mathrm{PKC}-2$ and $\mathrm{PKC} \imath / \mathrm{PKC}-3$ as candidate substrates for TORC2, whose activities are diminished by semaphorin input. Thus, we consider that the semaphorin signal is conveyed into destabilization of actin architecture through inhibition of the TORC2-PKC pathway. In agreement with this, we and others demonstrated that activationinactivation cycling and de novo synthesis of cofilin, an actin-depolymerizing factor, underlie the semaphorin-induced morphological events $^{7,8,44}$. In motile Dictyostelium, TORC2 regulation is confined to the leading edges of cAMP-oriented cells, where cytoskeletonrelated proteins are dynamically regulated by TORC2 (refs 42,45 ). It is interesting to speculate that semaphorin-induced inhibition of the TORC2-PKC pathway attenuates the rate of F-actin formation at the R1.p-R2.p boundary. Concertedly, semaphorin increases TORC1 and thereby translationally activates cytoskeleton-relevant proteins like cofilin, which enables acceleration of actin depolymerization locally at the cell-cell contact sites and allows for the proper positioning of ray 1 .

As our results and a previous report illustrate ${ }^{46}$, the choice of TOR partners between Raptor and Rictor seems to be controlled by physiological stimuli, serving as an important step in the regulation of the two TORCs signalling pathways. The precise molecular mechanism by which TORC assembly is regulated remains to be elucidated at present. We found that Raptor and Rictor are in a competitive relationship for binding to TOR, though the results obtained with overexpressing transgenes might not strictly reflect physiological conditions. This may imply that semaphorin mediates conversion of TORC2 to TORC1 simply by either destabilizing TORC2 or stabilizing TORC1 through biochemical modulation of the TORC components. In mammalian cells, competitive binding is implicated, at least partly, in FoxO-mediated regulation of assembly of mTOR complexes ${ }^{46}$. Otherwise, localization of the TORC components might be the basis for semaphorin-induced shift of the TOR adaptors. Recently, several reports have demonstrated the importance of changes in the subcellular localization of two TORCs on their functionalities. Rag GTPase-bound TORC1 is recruited from dispersed puncta to endomembrane structure on nutritional stimuli, where it is activated by Rheb ${ }^{47}$. In endothelial cells, Syndecan-4, a membrane-bound proteoglycan, targets TORC2 to membrane rafts, thereby rendering TORC 2 active but TORC 1 inactive ${ }^{48}$. It might be possible that semaphorin delocalizes and thus inactivates TORC2 while relocalizing TORC1 to its active sites. In the future, analysis of the subcellular localization of TOR and/or the TOR adaptors within ray precursor cells may provide more clues to the mechanism of the semaphorin action on TOR.
In conclusion, we have demonstrated genetic and biochemical evidence of TOR partnering by semaphoring-plexin signalling during cellular morphogenesis in C. elegans. Semaphorin-activated TORC1 augments translation through eIF4F as well as eIF2 activation, and concomitant TORC2 inhibition probably results in F-actin destabilization (Fig. 7). Therefore, we propose that TOR has a central role in integrating upstream semaphorin input and subsequently transducing the signal into divergent multiple downstream cascades.

\section{Methods}

Strains. C. elegans culture and genetics were performed as described by Brenner ${ }^{49}$. To facilitate the analyses of males, all strains carried him-5(e1490) or him-8(e1489). The following alleles were used in this study: [LGI] smp-1(ev715), smp-2(ev709), daf-16(mu86); [LGII] rict-1(nc41), rrf-3(pk1426), ncIs13[ajm-1::gfp]; [LGIII] aak-1(tm1944), strd-1(ok2283), rsks-1(tm1714); [LGIV] plx-1(nc37), tpa-1(k529), him-8(e1489); [LGV] pkc-1(nj1), rde-1(km56), par-4(it57), him-5(e1490); [LGX] pkc-2(ok328), aak-2(ok524), aakb-1(tm2658), ncIs35[lin-32p:: rde-1::egfp]; Bristol N2 and Hawaiian CB4856.

Isolation and characterization of rict-1(nc41) mutants. Suppressor screening was performed through mutagenesis on plx-1(nc37); him-5(e1490) mutants with ethyl methanesulfonate ${ }^{7}$. One isolated suppressor, $n c 41$, was out-crossed more than ten times. $n c 41$ single mutants were fertile and appeared healthy, but the brood size was reduced to $60 \%$ of wild-type N2 (N2: $275.8 \pm 25(n=4) ; n c 41: 161.7 \pm 25$ $(n=12))$.

Two-factor and three-factor crosses mapped $n c 41$ at about 8 map units right to rol-1 and about 9 map units left to $u n c-52$ on linkage group II. Further mapping was performed by utilizing SNPs between N2 and CB4856 strains. Using genomic DNA from 169 populations, nc41 was mapped between nucleotide (nt:) 12,398 on Y81G3A and nt: 18,109 on F32A11 (about $56 \mathrm{kbp}$ ). PCR using genomic DNA from $n c 41$ mutant populations as template failed to amplify DNA fragments around nt: 2,969 on F29C12, indicating that this region was deleted in $n c 41$. Subsequent sequencing revealed that $n c 41$ was a deletion between nt: 13,469 and nt: 13,938 (470 bp) within F29C12.3 gene (rict-1).

RNA interference. A cDNA fragment of plx-1 (nt: 603-1,912), rict-1 (nt: 1-892), daf-15 (nt: 1-555), let-363 (nt: 1-536), CeLST8/C10H11.8 (nt: 9-1,130), pkc-3 (nt: 10-1,780), akt-1 (nt: 5-747), akt-2 (nt: 18-1,387), spn-2 (nt: 29-1,077), or a genomic DNA fragment of ifg-1 (M110: 15,908-16,949) was subcloned into the pPD129.36 vector, and the resultant plasmids were transformed into the HT115 bacterial $\operatorname{strain}^{50}$. L4 hermaphrodites were grown on lawns of the transformed bacteria, and the F1 progeny were analysed for their phenotypes. Culture plates seeded with bacteria harbouring the empty pPD129.36 vector were used as controls. For the microscopic observation of rays, RNAi was performed in the background of $r r f-3(p k 1426)$, which gives hypersensitivity to RNAi ${ }^{51}$. For the ray-specific RNAi experiments, we used a line $r r f-3(p k 1426) ; r d e-1(k m 56) ; n c I s 35$ [lin-32p:: rde$1:: e g f p]$, where the RNAi-defective phenotype caused by $r d e-1$ mutation is rescued specifically in lin-32-expressing ray precursor cells ${ }^{52}$.

Immunoprecipitation. L3 and L4 males were collected in IP lysis buffer containing $40 \mathrm{mM}$ HEPES (pH7.5), $120 \mathrm{mM} \mathrm{NaCl}, 1 \mathrm{mM}$ EDTA and 0.3\% CHAPS supplemented with $1 \mathrm{mM}$ PMSF and protease inhibitor cocktail (Sigma), and dissolved by sonication (5-s sonication with a 5-s break, 10 cycles) and incubation with constant rotation for two hours at $4^{\circ} \mathrm{C}$. FLAG-tagged proteins were immunoprecipitated with anti-FLAG M2 affinity gel (Sigma) as per the manufacturer's instruction. To immunoprecipitate Myc- or HA-tagged proteins, lysates were precleared with protein G-agarose (Roche Applied Science) for two hours at $4{ }^{\circ} \mathrm{C}$, and incubated with anti-Myc (9E10) or anti-HA (Roche Applied Science) antibodies overnight at $4^{\circ} \mathrm{C}$. Samples were then incubated again with protein $\mathrm{G}$-agarose for another two hours at $4^{\circ} \mathrm{C}$, to allow for the precipitation. Immunoprecipitates were washed with CHAPS-free IP lysis buffer three times at $4^{\circ} \mathrm{C}$, and then eluted by boiling with $2 \times$ SDS sample buffer at $95^{\circ} \mathrm{C}$ for $3 \mathrm{~min}$. Both immunoprecipitates and lysates were subjected to subsequent western blot analysis.

Western blot analysis. Samples were run on 6 or 9\% SDS-PAGE and transferred to an Immobilon-P PVDF membrane (Millipore). Western blots were probed overnight at $4{ }^{\circ} \mathrm{C}$ with rabbit anti-FLAG (Sigma), mouse anti-Myc (9E10), mouse anti-HA (Roche), rabbit anti-phospho-4EBP1 (Cell Signaling Technology), rabbit anti-PKC $\alpha$ (Santa Cruz), rabbit anti-phospho-PKC $\alpha$ (Upstate), rabbit anti-eIF2 $\alpha^{7}$, or rabbit anti-phospho-eIF2 $\alpha$ (Cell Signaling) primary antibodies. The blots were visualized by incubation for two hours at room temperature with HRP-conjugated anti-mouse or anti-rabbit IgG secondary antibodies (Cell Signaling), followed by detection with an Immobilon Western chemiluminescent HRP substrate (Millipore). Images were captured and quantified with Luminescent Image Analyser LAS-4000 (Fujifilm). Paired $t$-test was used to evaluate differences in the signal intensities. 
Heat-shock-induced gene expression. L3 and L4 males carrying the extrachromosomal $h s p:: p l x-1$ and/or $h s p:: s m p-1$ transgenes on NGM plates were heatshocked for $15 \mathrm{~min}$ at $37^{\circ} \mathrm{C}$. Animals were collected two hours after heat-shock and subjected to Western blot analysis.

cDNA construction. Full-length wild-type rict-1 cDNA was constructed by fusing the $5^{\prime}$ RACE product corresponding to the 1 st-12th exons with the $3^{\prime}$ cDNA $y k 1311 f 01$ covering the ninth exon through $3^{\prime} \mathrm{UTR}$ of the gene, which was kindly provided by Yuji Kohara. To clone nc41-type rict- 1 cDNA, we extracted poly(A) RNAs from $n c 41$ mutants, reverse-transcribed the mRNAs using oligo-d(T) primer, and identified opal codon at the position 1,200th in the mutant rict-1 cDNA. Then, $3^{\prime}$ moiety of the mutant cDNA was PCR-amplified and digested with $B s p \mathrm{EI} / E c o \mathrm{RV}$ enzymes, with which the equivalent region of the wild-type rict-1 cDNA was replaced. Full-length wild-type daf- $15 \mathrm{cDNA}$ was constructed by fusing the $5^{\prime}$ RACE product corresponding to the 1 st-5th exons with $y k 1462$ covering the $3 \mathrm{rd}-12$ th exons and $y k 893$ covering the eighth exon through $3^{\prime}$ UTR of the gene. Full-length wild-type let-363 cDNA was constructed by fusing the $5^{\prime}$ RACE product with the $3^{\prime} \mathrm{cDNA} y k 22 f 1$. These cDNAs were modified to be flanked by $K p n I$ sites at both ends to facilitate the subsequent subcloning. Primer sequences for the generation of these cDNAs are available on request.

Generation of transgenic lines. The pPD49.78 and the pPD30.38 vectors were used to express genes of interest under the heat-shock promoter (hsp16-2) and the unc-54 promoter, respectively. The lin-32 promoter-inserted pPD49.26 vector was used to express genes in ray precursor cells ${ }^{7,28}$. FLAG, Myc, or HA adaptor sequence was fused to the $5^{\prime}$ end of cDNAs. The epitope-tagged daf-15 and rict-1 genes placed under the lin-32p are suggested to be functional, because they exerted the same effects on ray morphogenesis as their corresponding untagged ones (Supplementary Table S1). Each of the following DNAs flanked by synthetic restriction sites was inserted into the multiple cloning sites of each vector. The transgene harbouring cDNA for $p l x-1, \operatorname{smp}-1$, rict-1 (with or without a HA tag), daf-15 (with or without a Myc tag), let-363 (FLAG-tagged), human 4EBP1 (FLAG-tagged), or rat PKC $\alpha$ (FLAG-tagged) was mixed with pRF4 and injected into gonads of $\mathrm{N} 2$ hermaphrodites to establish stable extrachromosomal arrays $\mathrm{s}^{53}$ The lin-32p:: rde-1::egfp transgene was injected together with pRF4 to establish an extrachromosomal array, which was then chromosomally integrated by $\gamma$-ray irradiation $^{54}$ to generate $n c I s 35$.

Extrachromosomal arrays used and the final concentrations of the transgenes for injection are listed on Supplementary Table S2. Primer sequences for the generation of the transgenes are available on request.

Microscopy. Animals were mounted on $4 \%$ agar pads containing $1 \mathrm{mM}$ levamisole for immobilization. Ray phenotypes at the adult stage were analysed with Nomarski differential interference optics (ZEISS Axioplan 2). Images were captured with a Penguin 600CL CCD camera (Pixera). GFP expressions at the cell boundaries of epidermal ray precursor cells in $n c I s 13$ were scored and captured with a confocal microscopy Olympus Fluoview300.

Phenotypic analysis of ray 1. Three 'levels' (Level 1, Level 2, Level 3) were used as criteria for the quantitative evaluation of the ray 1 phenotype ${ }^{7}$. Briefly, level 1 refers to a normal state where ray 1 is closely situated to its neighbouring ray 2 . Level 2 refers to a mildly defective state where ray 1 is separated from ray 2 within a cuticular fan. Level 3 refers to a severely defective state where ray 1 is situated apart from ray 2 outside of a fan. Fisher's exact test was used to statistically evaluate differences in the ray 1 phenotype between two genotypes (Supplementary Table S1).

\section{References}

1. Kruger, R. P., Aurandt, J. \& Guan, K. L. Semaphorins command cells to move. Nat. Rev. Mol. Cell Biol. 6, 789-800 (2005).

2. Negishi, M., Oinuma, I. \& Katoh, H. Plexins: axon guidance and signal transduction. Cell Mol. Life Sci. 62, 1363-1371 (2005).

3. Tran, T. S., Kolodkin, A. L. \& Bharadwaj, R. Semaphorin regulation of cellular morphology. Annu. Rev. Cell Dev. Biol. 23, 263-292 (2007).

4. Hershey, J. W. B. \& Merrick, W. C. in Translational Control of Gene Expression (eds Sonenberg, N., Hershey, J. W. B. \& Mathews, M. B.) 33-88 (Cold Spring Harbor Laboratory Press, 2000).

5. Campbell, D. S. \& Holt, C. E. Chemotropic responses of retinal growth cones mediated by rapid local protein synthesis and degradation. Neuron 32, 1013-1026 (2001)

6. Wu, K. Y. et al. Local translation of RhoA regulates growth cone collapse. Nature 436, 1020-1024 (2005)

7. Nukazuka, A., Fujisawa, H., Inada, T., Oda, Y. \& Takagi, S. Semaphorin controls epidermal morphogenesis by stimulating mRNA translation via eIF2alpha in Caenorhabditis elegans. Genes Dev. 22, 1025-1036 (2008).

8. Piper, M. et al. Signaling mechanisms underlying Slit2-induced collapse of Xenopus retinal growth cones. Neuron 49, 215-228 (2006).
9. Guertin, D. A. \& Sabatini, D. M. Defining the role of mTOR in cancer. Cancer Cell 12, 9-22 (2007).

10. Polak, P. \& Hall, M. N. mTOR and the control of whole body metabolism. Curr Opin. Cell Biol. 21, 209-218 (2009).

11. Kim, D. H. et al. mTOR interacts with raptor to form a nutrient-sensitive complex that signals to the cell growth machinery. Cell 110, 163-175 (2002).

12. Hara, K. et al. Raptor, a binding partner of target of rapamycin (TOR), mediates TOR action. Cell 110, 177-189 (2002).

13. Sarbassov, D. D. et al. Rictor, a novel binding partner of mTOR, defines a rapamycin-insensitive and raptor-independent pathway that regulates the cytoskeleton. Curr. Biol. 14, 1296-1302 (2004).

14. Jacinto, E. et al. Mammalian TOR complex 2 controls the actin cytoskeleton and is rapamycin insensitive. Nat. Cell Biol. 6, 1122-1128 (2004).

15. Long, X., Lin, Y., Ortiz-Vega, S., Yonezawa, K. \& Avruch, J. Rheb binds and regulates the mTOR kinase. Curr. Biol. 15, 702-713 (2005).

16. Garami, A. et al. Insulin activation of Rheb, a mediator of mTOR/S6K/4E-BP signaling, is inhibited by TSC1 and 2. Mol. Cell 11, 1457-1466 (2003).

17. Inoki, K., Li, Y., Xu, T. \& Guan, K. L. Rheb GTPase is a direct target of TSC2 GAP activity and regulates mTOR signaling. Genes Dev. 17, 1829-1834 (2003).

18. Inoki, K., Zhu, T. \& Guan, K. L. TSC2 mediates cellular energy response to control cell growth and survival. Cell 115, 577-590 (2003).

19. Hay, N. \& Sonenberg, N. Upstream and downstream of mTOR. Genes Dev. 18, 1926-1945 (2004).

20. Hernandez-Negrete, I. et al. P-Rex1 links mammalian target of rapamycin signaling to Rac activation and cell migration. J. Biol. Chem. 282, 23708-23715 (2007).

21. Sarbassov, D. D., Guertin, D. A., Ali, S. M. \& Sabatini, D. M. Phosphorylation and regulation of Akt/PKB by the rictor-mTOR complex. Science 307, 1098-1101 (2005).

22. Shiota, C., Woo, J. T., Lindner, J., Shelton, K. D. \& Magnuson, M. A. Multiallelic disruption of the rictor gene in mice reveals that mTOR complex 2 is essential for fetal growth and viability. Dev. Cell 11, 583-589 (2006).

23. Guertin, D. A. et al. Ablation in mice of the mTORC components raptor, rictor or mLST8 reveals that mTORC2 is required for signaling to Akt-FOXO and PKCalpha, but not S6K1. Dev. Cell 11, 859-871 (2006).

24. Fujii, T. et al. Caenorhabditis elegans PlexinA, PLX-1, interacts with transmembrane semaphorins and regulates epidermal morphogenesis. Development 129, 2053-2063 (2002).

25. Ginzburg, V. E., Roy, P. J. \& Culotti, J. G. Semaphorin 1a and semaphorin $1 \mathrm{~b}$ are required for correct epidermal cell positioning and adhesion during morphogenesis in C. elegans. Development 129, 2065-2078 (2002).

26. Emmons, S. W. in WormBook (ed. WormBook The C. elegans Research Community) (http://www.wormbook.org 2005)

27. Baird, S. E., Fitch, D. H., Kassem, I. A. \& Emmons, S. W. Pattern formation in the nematode epidermis: determination of the arrangement of peripheral sense organs in the C. elegans male tail. Development 113, 515-526 (1991).

28. Portman, D. S. \& Emmons, S. W. The basic helix-loop-helix transcription factors LIN-32 and HLH-2 function together in multiple steps of a C. elegans neuronal sublineage. Development 127, 5415-5426 (2000).

29. Soukas, A. A., Kane, E. A., Carr, C. E., Melo, J. A. \& Ruvkun, G. Rictor/TORC2 regulates fat metabolism, feeding, growth, and life span in Caenorhabditis elegans. Genes Dev. 23, 496-511 (2009)

30. Jones, K. T., Greer, E. R., Pearce, D. \& Ashrafi, K. Rictor/TORC2 regulates caenorhabditis elegans fat storage, body size, and development through sgk-1. PLoS Biol. 7, e60 (2009).

31. Long, X. et al. TOR deficiency in C. elegans causes developmental arrest and intestinal atrophy by inhibition of mRNA translation. Curr. Biol. 12, 1448-1461 (2002).

32. Jia, K., Chen, D. \& Riddle, D. L. The TOR pathway interacts with the insulin signaling pathway to regulate C. elegans larval development, metabolism and life span. Development 131, 3897-3906 (2004).

33. Hardie, D. G. New roles for the LKB1 $\rightarrow$ AMPK pathway. Curr. Opin. Cell Biol. 17, 167-173 (2005)

34. Watts, J. L., Morton, D. G., Bestman, J. \& Kemphues, K. J. The C. elegans par-4 gene encodes a putative serine-threonine kinase required for establishing embryonic asymmetry. Development 127, 1467-1475 (2000).

35. Apfeld, J., O’Connor, G., McDonagh, T., DiStefano, P. S. \& Curtis, R. The AMP activated protein kinase AAK-2 links energy levels and insulin-like signals to lifespan in C. elegans. Genes Dev. 18, 3004-3009 (2004).

36. Narbonne, P. \& Roy, R. Inhibition of germline proliferation during C. elegans dauer development requires PTEN, LKB1 and AMPK signalling. Development 133, 611-619 (2006).

37. Li, W., DeBella, L. R., Guven-Ozkan, T., Lin, R. \& Rose, L. S. An eIF4E-binding protein regulates katanin protein levels in C. elegans embryos. J. Cell Biol. 187, 33-42 (2009)

38. Tabuse, Y. Protein kinase C isotypes in C. elegans. J. Biochem. 132, 519-522 (2002).

39. Paradis, S. \& Ruvkun, G. Caenorhabditis elegans Akt/PKB transduces insulin receptor-like signals from AGE-1 PI3 kinase to the DAF-16 transcription factor. Genes Dev. 12, 2488-2498 (1998). 
40. Cherkasova, V. A. \& Hinnebusch, A. G. Translational control by TOR and TAP42 through dephosphorylation of eIF2alpha kinase GCN2. Genes Dev. 17, 859-872 (2003).

41. Kubota, H., Obata, T., Ota, K., Sasaki, T. \& Ito, T. Rapamycin-induced translational derepression of GCN4 mRNA involves a novel mechanism for activation of the eIF2 alpha kinase GCN2. J. Biol. Chem. 278, 20457-20460 (2003).

42. Kamimura, Y. et al. PIP3-independent activation of TorC2 and PKB at the cell's leading edge mediates chemotaxis. Curr. Biol. 18, 1034-1043 (2008).

43. Liu, L., Das, S., Losert, W. \& Parent, C. A. mTORC2 regulates neutrophil chemotaxis in a cAMP- and RhoA-dependent fashion. Dev. Cell 19, 845-857 (2010).

44. Aizawa, H. et al. Phosphorylation of cofilin by LIM-kinase is necessary for semaphorin 3A-induced growth cone collapse. Nat. Neurosci. 4, 367-373 (2001)

45. Charest, P. G. et al. A Ras signaling complex controls the RasC-TORC2 pathway and directed cell migration. Dev. Cell 18, 737-749 (2010).

46. Chen, C. C. et al. FoxOs inhibit mTORC1 and activate Akt by inducing the expression of Sestrin3 and Rictor. Dev. Cell 18, 592-604 (2010).

47. Sancak, Y. et al. The Rag GTPases bind raptor and mediate amino acid signaling to mTORC1. Science 320, 1496-1501 (2008).

48. Partovian, C., Ju, R., Zhuang, Z. W., Martin, K. A. \& Simons, M. Syndecan-4 regulates subcellular localization of mTOR Complex2 and Akt activation in a PKCalpha-dependent manner in endothelial cells. Mol. Cell 32, 140-149 (2008).

49. Brenner, S. The genetics of Caenorhabditis elegans. Genetics 77, 71-94 (1974).

50. Timmons, L. \& Fire, A. Specific interference by ingested dsRNA. Nature 395, 854 (1998).

51. Simmer, F. et al. Loss of the putative RNA-directed RNA polymerase RRF-3 makes C. elegans hypersensitive to RNAi. Curr. Biol. 12, 1317-1319 (2002).

52. Qadota, H. et al. Establishment of a tissue-specific RNAi system in C. elegans. Gene 400, 166-173 (2007)

53. Mello, C. \& Fire, A. DNA transformation. Methods Cell Biol. 48, 451-482 (1995).

54. Shioi, G. et al. Mutations affecting nerve attachment of Caenorhabditis elegans Genetics 157, 1611-1622 (2001).

\section{Acknowledgements}

We thank the staff of Fujisawa, Oda and Matsumoto Laboratories, Naoki Hisamoto, Hiromi Hirata, and Fumikazu Suto for discussion and comments; Motoshi Suzuki for constructing $n c I s 35$ [lin-32p :: rde-1::egfp]; Yuji Kohara for cDNA clones; Andrew Fire for pPD vectors; Naoki Hisamoto for $r d e-1(\mathrm{~km} 56)$ mutants; Hiroaki Imataka for human 4EBP1 cDNA; and Yoshitaka Ono for rat PKC $\alpha$ cDNA. Some strains were provided by the Caenorhabditis Genetic Center, which is funded by the National Institute for Health National Center for Research Resources, and National Bioresource Project funded by the Ministry of Education, Culture, Sports, Science and Technology of Japan. This work was supported by grants from the Ministry of Education, Culture, Sports, Science and Technology of Japan (Y.O., H.F., S.T.), a grant from CREST (Core Research for Evolutional Science and Technology) of the Japan Science and Technology Corporation (H.F.), and grants from The Sumitomo Foundation and The Naito Foundation (S.T.) A.N. is grateful to the GCOE Program of Nagoya University for support.

\section{Author contributions}

A.N. performed all the experiments, except for the cloning of cDNAs for rict- 1 and daf- 15 which was conducted by S.T. A.N. designed the study and wrote the manuscript. K.M., Y.O., H.F. and S.T. directed the study and wrote the manuscript.

\section{Additional information}

Supplementary Information accompanies this paper at http://www.nature.com/ naturecommunications

Competing financial interests: The authors declare no competing financial interests.

Reprints and permission information is available online at http://npg.nature.com/ reprintsandpermissions/

How to cite this article: Nukazuka, A. et al. A shift of the TOR adaptor from Rictor towards Raptor by semaphorin in C. elegans. Nat. Commun. 2:484 doi: $10.1038 /$ ncomms1495 (2011)

License: This work is licensed under a Creative Commons Attribution-NonCommercialShare Alike 3.0 Unported License. To view a copy of this license, visit http:// creativecommons.org/licenses/by-nc-sa/3.0/ 